\title{
CARACTERIZAÇÃO CLÍNICA E EPIDEMIOLÓGICA DE PACIENTES ATENDIDOS POR UM PROGRAMA PÚBLICO DE ATENÇÃO DOMICILIAR
}

Daniel Vinicius Alves Silva ${ }^{1}$, Júlia Rocha do Carmo ${ }^{3}$, Monique Évellin Alves Cruz², Carolina Amaral Oliveira Rodrigues ${ }^{3}$, Edileuza Teixeira Santana ${ }^{3}$, Diego Dias de Araújo 3

Objetivo: caracterizar clínica e epidemiologicamente os pacientes atendidos por um programa público de atenção domiciliar na cidade de Montes Claros, Minas Gerais, Brasil. Metodologia: Estudo transversal e exploratório, realizado entre junho de 2017 e janeiro de 2018, com 131 pacientes cadastrados e atendidos pelo Serviço de Atenção Domiciliar - Melhor em Casa. Para o levantamento dos dados utilizou-se um instrumento contendo variáveis sociodemográficas, clínicas e funcionais, analisados por estatística descritiva. Resultados: A maioria dos pacientes eram idosos (67,9\%), do sexo feminino (55\%), pardos (47,3\%), casados (31,3\%) e/ou solteiros (31,3\%), restritos ao leito (71\%) e alimentavam-se via oral (68,7\%). As doenças vasculares (42\%) foram as mais prevalentes, $45,8 \%$ dos pacientes apresentavam lesão por pressão e o atendimento domiciliar foi realizado principalmente pelo enfermeiro (83,2\%) e médico (82,4\%). Conclusão: A caracterização do perfil, além de descrever problemas, contribui para o cuidado do paciente, impactando no planejamento e implementação de intervenções adequadas.

Descritores: Pacientes Domiciliares; Serviços de Assistência Domiciliar; Perfil de Saúde; Idoso.

\section{CLINICALAND EPIDEMIOLOGICAL CHARACTERIZATION OF PATIENTS TREATED BY A PUBLIC HOME CARE PROGRAM}

Objective: to characterize clinically and epidemiologically the patients attended by a public home care program in the city of Montes Claros, Minas Gerais, Brazil. Methodology: A cross - sectional and exploratory study was carried out between June 2017 and January 2018, with 131 patients enrolled and attended by the Home Care Service - Better at Home. Data were collected using an instrument containing sociodemographic, clinical and functional variables and were analyzed by descriptive statistics. Results: The majority of the patients were elderly (67.9\%), female (55\%), brown (47.3\%), married (31.3\%) and / or unmarried to bed (71\%) and were fed orally (68.7\%). Vascular diseases (42\%) were the most prevalent, $45.8 \%$ of the patients had pressure lesions, and home care was performed mainly by the nurse (83.2\%) and the physician ( $82.4 \%)$. Conclusion: The characterization of the profile, besides describing problems, contributes to patient care, impacting on the planning and implementation of appropriate interventions.

Descriptors: Homebound Persons; Home Care Services; Health Profile; Aged.

\section{CARACTERIZACIÓN CLÍNICA Y EPIDEMIOLÓGICA DE PACIENTES ATENDIDOS POR UN PROGRAMA PÚBLICO DE ATENCIÓN DOMICILIARIA}

Objetivo: caracterizar clínica y epidemiológicamente a los pacientes atendidos por un programa público de atención domiciliaria en la ciudad de Montes Claros, Minas Gerais, Brasil. Metodología: Estudio transversal y exploratorio, realizado entre junio de 2017 y enero de 2018, con 131 pacientes catastrados y atendidos por el Servicio de Atención Domiciliar - Mejor en Casa. Para el levantamiento de los datos se utilizó un instrumento que contenía variables sociodemográficas, clínicas y funcionales, analizados por estadística descriptiva. Resultados: La mayoría de los pacientes eran ancianos (67,9\%), del sexo femenino (55\%), pardos (47,3\%), casados (31,3\%) y / o solteros (31,3\%), restringidos al lecho (71\%) y se alimentaban vía oral (68,7\%). Las enfermedades vasculares (42\%) fueron las más prevalentes, el 45,8\% de los pacientes presentaban lesión por presión y la atención domiciliaria fue realizada principalmente por el enfermero (83,2\%) y médico $(82,4 \%)$. Conclusión: La caracterización del perfil, además de describir problemas, contribuye al cuidado del paciente, impactando en la planificación e implementación de intervenciones adecuadas.

Descriptores: Personas Imposibilitadas; Servicios de Atención de Salud a Domicilio; Anciano. 


\section{INTRODUCÃO}

Nas últimas décadas, o Brasil vem passando por uma série de mudanças como o envelhecimento populacional, a transição epidemiológica e a crise do modelo de atenção à saúde. Diante desta realidade, foi necessário buscar alternativas efetivas para atender às necessidades de saúde da população(1,2)

Assim, desenvolveu-se no país diferentes estratégias de cuidado em saúde com destaque para aquelas com atividades próximas ao domicílio do paciente, como a Estratégia de Saúde da Família, os serviços de Atenção Domiciliar (AD), propostas de cuidados paliativos domiciliares, entre outros ${ }^{(3,4)}$.

O termo "assistência domiciliar" vem do inglês home care e é definido como cuidado no lar. Compreende um conjunto de procedimentos hospitalares passiveis de serem desenvolvidos no domicílio do paciente ${ }^{(5)}$. No Brasil, a Política Nacional de Atenção Domiciliar (PNAD) foi instituida pela Portaria $n^{\circ}$ 2.029 de 24 de agosto de 2011 e regulamenta a atenção domiciliar por meio do Programa Melhor em Casa (PMC), sendo regida atualmente pela Portaria $n=825$ de 2016 (6.7). AAD é coordenada pelos Serviços de Atenção Domiciliar (SAD) e inclui-se na Rede de Atenção à Saúde (RAS) do Sistema Único de Saúde (SUS). Apropriando dos princípios e diretrizes do SUS, devem ser observados na organização da SAD a universalidade, equidade, a integralidade, a resolubilidade e a ampliação do acesso, associados ao acolhimento e à humanização(8,9)

A AD é caracterizada por desenvolver ações de promoção à saúde, prevenção, tratamento de doenças, paliação e reabilitação das condições de saúde assistidas em domicílio $^{(6,10)}$. Essa modalidade assistencial é indicada a pacientes em situação de restrição ao leito ou ao lar, bem como, para atender às necessidades de pessoas dependentes ou com doenças crônicas. Na AD, destacam-se entre as condições de indicação, as sindromes demenciais e de fragilidade, acidente cerebrovascular, doenças cardiocirculatórias, respiratórias e osteomusculares, sequelados de acidentes automobilísticos e violência(6)

Enfatiza-se que AD preza por valores como a convivência familiar, afeto, reintegração do usuário ao núcleo familiar e de apoio, bem como de uma presumida melhor qualidade de vida do paciente; também, corrobora para redução de custos e otimização dos leitos hospitalares ${ }^{(5,11)}$

Neste contexto, o presente estudo teve como objetivo caracterizar clínica e epidemiologicamente os pacientes atendidos por um programa público de atenção domiciliar na cidade de Montes Claros, Minas Gerais, Brasil.

\section{METÓDOS}

\section{Tipo de estudo}

Trata-se de um estudo exploratório descritivo, do tipo transversal.

\section{Participantes da pesquisa}

A população alvo desta pesquisa constituiu-se por 172 pacientes cadastrados e em atendimento pelo PMC. Os critérios de inclusão foram: idade igual ou superior a 18 anos, estar cadastrado e em atendimento pelo PMC e consentir em participar do estudo ou ter sua participação autorizada pelo responsável/cuidador por meio da assinatura do Termo de Consentimento Livre e Esclarecido (TCLE) ou pelo Termo de Assentimento, respectivamente. Já os critérios de exclusão foram: não ser encontrado no domicílio em até três tentativas, evoluísse ao óbito antes que fosse realizada a coleta de dados e pacientes em internação hospitalar.

Assim, após aplicação dos critérios de inclusão e exclusão, dos 172 pacientes, treze foram excluidos por evoluíram ao óbito antes que fosse realizada a coleta de dados, dez por serem menores de idade, sete por não serem encontrados no domicilio em até três tentativas, seis por estarem em internação hospitalar e cinco por não consentirem a participação no estudo. Após aplicação dos critérios de inclusão e exclusão, contou-se com um universo de 131 pacientes.

\section{Local do estudo}

Este estudo foi desenvolvido com pacientes cadastrados e atendidos pelo SAD - Melhor em Casa, do município de Montes Claros, Minas Gerais, Brasil, no período de junho de 2017 a janeiro de 2018. Esse período foi o necessário para se realizar a coleta de dados de todos os pacientes cadastrados e atendidos pelo PMC.

O PMC presta assistência multiprofissional adequada e gratuita a pacientes com problemas de pequena a média complexidade. Atualmente, conta com quatro equipes multiprofissionais de atenção domiciliar, compostas por médico, enfermeiro, técnico em enfermagem e fisioterapeuta, além de duas equipes multiprofissionais de apoio, integradas por fonoaudiólogo, nutricionista, assistente social, odontólogo, psicólogo, terapeuta ocupacional e farmacêutico.

\section{Coleta dos dados}

Para coleta de dados, utilizou-se um instrumento sistematizado, elaborado pelos pesquisadores a partir da experiência profissional e da revisão da literatura(5,12-13,15). O instrumento incluía variáveis de caracterização socioeconômica, clínica e funcionais, a saber: nome, idade, sexo, raça, estado civil, diagnósticos médicos, grau de dependência para deambular, via de respiração, via de alimentação, suporte de oxigênio suplementar, presença de lesão por pressão (LPP), local da lesão.

O procedimento de coleta de dados foi realizado no domicílio dos pacientes por um graduando em enfermagem, do sé- 
timo período do curso de graduação em enfermagem de uma universidade pública do estado de Minas Gerais, devidamente capacitado e sob supervisão do professor orientador.

\section{Procedimentos de análise dos dados}

No tratamento dos dados foi realizada dupla digitação no programa software Statistical Package for Social Sciente (SPSS), versão 20.0, e após verificação da consistência, os dados foram analisados por estatística descritiva (frequências simples e percentual).

\section{Procedimentos éticos}

Este estudo respeita os aspectos éticos da Resolução 466/2012 e foi aprovado pelo Comitê de Ética em Pesquisa da Universidade Estadual de Montes Claros, sob o protocolo CAAE-62123716.3.0000.5146.

\section{RESULTADOS}

Entre os 131 pacientes, destaca-se o sexo feminino $(n=72$; $55 \%)$, raça parda ( $n=62 ; 47,3 \%)$, casados ( $n=41 ; 31,3 \%$ ) e/ou solteiros ( $n=41 ; 31,3 \%)$ e idosos ( $n=89 ; 67,9 \%)$. Na faixa etária, a maior frequência foi entre 60-79 anos ( $n=46 ; 35,1 \%)$, média de idade 66,8 anos $( \pm 21,2)$ e mediana 72 anos (Tabela 1 ).

Tabela 1. Características sociodemográficas dos pacientes em atendimento domiciliar. Montes Claros, MG, junho de 2017 a janeiro de 2018.

\begin{tabular}{lcc}
\multicolumn{1}{l}{ Variáveis } & $\mathbf{N}=131$ & $\%$ \\
\hline Sexo & 72 & 55 \\
Feminino & 59 & 45 \\
\hline Masculino & & \\
\hline Raça & 51 & 38,9 \\
\hline Branco & 16 & 12,2 \\
\hline Negro & 62 & 47,3 \\
\hline Pardo & 2 & 1,5 \\
\hline Amarelo & & \\
\hline Estado civil & 41 & 31,3 \\
\hline Casado & 41 & 31,3 \\
\hline Solteiro & 3 & 2,3 \\
\hline União estável & 7 & 5,3 \\
\hline Divorciado & 39 & 29,8 \\
\hline Viúvo & & \\
\hline Faixa etária (anos) & 24 & 18,3 \\
\hline 18-39 & 18 & 13,8 \\
\hline $40-59$ & 46 & 35,1 \\
\hline 60-79 & 40 & 30,5 \\
\hline 80-99 & 3 & \\
\hline Acima de 99 & & \\
\hline
\end{tabular}

Fonte: Pesquisa de Campo, junho de 2017 a janeiro de 2018.
Em relação à classe do diagnóstico médico de indicação para $A D$, as doenças mais prevalentes foram agrupadas em vasculares ( $n=55 ; 42 \%)$, neurológicas ( $n=36 ; 27,5 \%)$ e metabólicas ( $n=26 ; 19,8 \%$ ) (Tabela 2).

Tabela 2. Classes de diagnóstico médico para indicação de atenção domiciliar identificadas em pacientes de atendimento domiciliar. Montes Claros, MG, junho de 2017 a janeiro de 2018.

\begin{tabular}{lcc} 
Classes de diagnóstico médico & $\mathbf{N}$ & $\%$ \\
\hline Doença Vascular & 55 & 42,0 \\
\hline Doença Neurológica & 36 & 27,5 \\
\hline Doença Metabólica & 26 & 19,8 \\
\hline Trauma & 21 & 16,0 \\
\hline Doença oncológica & 19 & 14,5 \\
\hline Doença Infecciosa & 13 & 9,9 \\
\hline Doença Cardiaca & 10 & 7,6 \\
\hline Doença Músculo-esquelética & 9 & 6,9 \\
\hline Doença Mental & 5 & 3,8 \\
\hline Doença Pulmonar & 5 & 3,8 \\
Outros & 26 & 19,8
\end{tabular}

Fonte: Pesquisa de Campo, junho de 2017 a janeiro de 2018.

Conforme o diagnóstico médico específico para indicação de atendimento na AD, o Acidente Vascular Encefálico (AVE) foi o mais prevalente, sendo diagnosticado em $26,7 \% \quad(n=35)$ dos pacientes.

As comorbidades identificadas com maior frequência nos pacientes da AD foram a Hipertensão Arterial Sistêmica (HAS) ( $n=33 ; 25,2 \%)$, Alzheimer ( $n=22 ; 16,8 \%)$, Diabetes Mellitus (DM) $(n=22 ; 16,8 \%)$ e Neoplasias ( $n=19 ; 14,5 \%)$. Destaca-se que 62 (47,3\%) pacientes estavam em uso de polifarmácia, ou seja, cinco ou mais medicamentos por dia.

As características clínicas e funcionais evidenciam que 93 (71\%) pacientes encontravam-se restritos ao leito e 16 (12,2\%) pacientes deambulavam sem dependência. Acerca da via de alimentação, 90 (68,7\%) pacientes se alimentavam via oral e $24(18,3 \%)$ via gastrostomia. A maioria dos pacientes respirava em ar ambiente e 10 (7,6\%) utilizavam oxigênio. Dos 131 pacientes, 60 (45,8\%) apresentavam LPP, sendo o local mais acometido a região sacral ( $n=43 ; 32,8 \%$ ) (Tabela 3).

Tabela 3. Características clínicas e funcionais dos pacientes em atendido domiciliar. Montes Claros, MG, junho de 2017 a janeiro de 2018 .

\begin{tabular}{lcc}
\multicolumn{1}{c}{ Variáveis } & N=131 & $\%$ \\
\hline Niveis de dependência & & \\
\hline Não deambula (restrito ao leito) & 93 & 71,0 \\
\hline Deambula com dependência/cadeirante & 22 & 16,8
\end{tabular}


Deambula sem dependência

16

12,2

\begin{tabular}{lcc} 
Via de alimentação & & \\
\hline Oral & 90 & 68,7 \\
\hline Gastrostomia & 24 & 18,3 \\
\hline Enteral & 14 & 10,7 \\
\hline Jejunostomia & 3 & 2,3 \\
\hline Uso de traqueostomia & & \\
\hline Não & 114 & 87 \\
\hline Sim & 17 & 13 \\
\hline Suporte de oxigênio suplementar & & \\
\hline Ar ambiente & 121 & 92,4 \\
\hline Oxigenoterapia & 10 & 7,6 \\
\hline Presença de lesão de pressão & & \\
\hline Sim & 60 & 45,8 \\
\hline Não & 71 & 54,2 \\
\hline Local da lesão & & \\
\hline Sacral & 43 & 32,8 \\
\hline Trocanteres & 11 & 8,4 \\
\hline Calcâneos & 9 & 6,9 \\
\hline Outros & 9 & 6,9 \\
\hline Sol & & \\
\hline
\end{tabular}

Fonte: Pesquisa de Campo, junho de 2017 a janeiro de 2018.

Verifica-se na Tabela 4 os dados referentes ao número de pacientes atendidos pela equipe multidisciplinar. Evidencia-se que os profissionais mais atuantes foram o enfermeiro $(n=109 ; 83,2 \%)$ e o médico ( $n=108 ; 82,4 \%)$.

Tabela 4. Número de pacientes atendidos pela equipe multidisciplinar. Montes Claros, MG, junho de 2017 a janeiro de 2018 .

\begin{tabular}{|c|c|c|}
\hline Equipe Multidisciplinar & $\mathrm{N}=131$ & $\%$ \\
\hline Enfermeiro & 109 & 83,2 \\
\hline Médico & 108 & 82,4 \\
\hline Fisioterapeuta & 96 & 73,3 \\
\hline Técnico de Enfermagem & 90 & 68,7 \\
\hline Nutricionista & 66 & 50,4 \\
\hline Psicólogo & 57 & 43,5 \\
\hline Fonoaudiólogo & 37 & 28,2 \\
\hline Assistente Social & 20 & 15,3 \\
\hline Odontólogo & 2 & 1,5 \\
\hline Terapeuta ocupacional & 1 & 0,8 \\
\hline Farmacêutico & 1 & 0,8 \\
\hline
\end{tabular}

Fonte: Pesquisa de Campo, junho de 2017 a janeiro de 2018.

\section{DISCUSSÃO}

Neste estudo, as características sociodemográficas dos pacientes mostraram-se semelhantes à literatura nacional(5,12,14-15) e internacional(16-17), em relação à população em as- sistência domiciliar. Os dados revelam que houve uma maior distribuição de pacientes do sexo feminino (55\%), compativeis com diversos estudos, que encontraram uma prevalência que variou entre $54,7 \%$ e $68 \%(5,12,14,16-17)$.

Em relação à idade, predominou a população idosa (67,9\%), com maior frequência na faixa etária de 60-79 anos (35,1\%). Estudo realizado na mesma cidade, em um programa hospitalar de internação domiciliar, identificou uma população semelhante, com $63,5 \%$ de idosos, sendo $37,2 \%$ na faixa etária entre 61-80 anos ${ }^{(12)}$. Já o estudo que buscou identificar o perfil de pacientes em atendimento domiciliar na cidade de Maceió, Alagoas, verificou que $80 \%$ são idosos e que a faixa etária de maior prevalência (44\%), foi de 79 anos ou mais ${ }^{(5)}$.

No presente estudo, a média de idade foi de 66,8 anos. Um estudo que avaliou prontuários de pacientes domiciliares de instituições privadas nos Estados Unidos, a média de idade foi de 62 anos $^{(16)}$. Em estudo multicêntrico, realizado nas cidades de Málaga, Costa do Sol, Almeria e Granada, localizadas na Espanha, a média de idade foi de 75,49 anos $^{(17)}$. O perfil de atendimento encontrado pode-se justificar pelo processo de transição demográfica que vem ocorrendo mundialmente e o Brasil acompanha essas tendências, como o aumento no número de idosos e o fenômeno de feminilização do envelhecimento, devido a maior expectativa de vida das mulheres em relação aos homens ${ }^{(2,18,19)}$.

Ressalta-se que com o avançar da idade há uma redução da reserva funcional, da capacidade do organismo adaptar-se para manter o equilibrio energético, o que pode comprometer os mecanismos necessários para a realização das atividades e aumentar a prevalência de doenças, agravos e incapacidades, tornando a pessoa idosa mais propensa a desenvolver a condição de fragilidade e consequentemente a necessidade de cuidados domiciliares ${ }^{(19)}$.

Neste estudo, as doenças vasculares foram predominantes (42\%), sendo o principal diagnóstico para indicação de AD o AVE (26,7\%). Evidencia-se semelhança com outros estudos em pacientes domiciliares ${ }^{(5,14,20)}$. Entretanto, há divergência com estudos ${ }^{(12,15)}$ em que a pneumonia foi o principal diagnóstico para indicação. A divergência nos perfis de doenças para indicação de AD pode estar associada às questões epidemiológicas de cada região e ao tipo de serviço de assistência domiciliar

Enfatiza-se que o AVE é a primeira causa de internação no Brasil; caracteriza-se por ser uma doença incapacitante que pode levar ao óbito; comumente resulta em sequelas físicas e mentais, restringindo a funcionalidade do indivíduo, principalmente nas atividades de vida diárias (AVDs) $)^{(5,21)}$. Neste estudo, além do AVE, outras doenças incapacitantes foram identificadas, com destaque para o Alzheimer, sindromes demenciais, Parkison, doenças osteomusculares e traumas. 
Verificou-se que a maioria dos pacientes nesta investigação apresentou mais de uma doença e consequentemente o uso de diversos medicamentos para o tratamento, sendo a polifarmácia identificada em $47,3 \%$ dos pacientes. No Brasil, $70 \%$ dos idosos têm pelo menos uma doença crônica que requer uma medicação para o tratamento e 60\% estão em uso de polifarmácia. $\mathrm{O}$ uso de múltiplos medicamentos concomitantemente está associado a desfechos negativos, como maior frequência de interação medicamentosa, perda de funcionalidade, quedas, fragilidade, desnutrição e até mesmo a morte ${ }^{(22)}$.

No que diz respeito à funcionalidade dos pacientes neste estudo, $71 \%$ eram pacientes com restrição ao leito. No estudo desenvolvido na cidade de Maceió, Alagoas ${ }^{(5)}$ verificou-se que, do total de pacientes, $72,5 \%$ eram restritos ao leito, sendo que $72,3 \%$ no serviço público e $72,7 \%$ no particular. Já em estudo conduzido em Belo Horizonte, Minas Gerais, identificou que $33,7 \%$ dos pacientes eram totalmente dependentes para $\mathrm{AVDs}^{(15)}$, consequentemente, restritos ao leito.

Com relação à via de alimentação, no presente estudo, $31,3 \%$ dos pacientes se alimentavam por outras vias que não a oral. A alimentação é condição essencial para a manutenção da vida humana, e é fundamental para a promoção, manutenção e/ou recuperação da saúde. O aumento do número de idosos na população, da prevalência de pessoas com doenças crônicas e de vítimas de acidentes de trânsito e de situações de violência podem ter como consequência alterações clínicas associadas à deglutição e/ou integridade do trato gastrointestinal(23). Nessa perspectiva, a terapia nutricional ganha enorme importância, tornando-se essencial nos cuidados implementados ao paciente domiciliar, impactando diretamente na evolução clínica bem como na redução da morbimortalidade.

Quanto ao uso de traqueostomia, no presente estudo, $13 \%$ dos pacientes encontravam-se com esse dispositivo e 7,6\% estavam em uso de oxigenoterapia. Estudo ${ }^{(5)}$ que comparou um serviço domiciliar público e dois privados, observou-se que 1,8\% dos pacientes atendidos pelo programa público utilizava traqueostomia em comparação a 16,9\% do serviço privado, sendo que 1,3 e 17,8\% usavam oxigenoterapia, respectivamente.

No que concerne à presença de LPP, evidenciou-se uma prevalência de $45,8 \%$, sendo a região mais acometida a sacral (32,8\%), seguida das regiões dos trocanteres $(8,4 \%)$, e calcâneos (6,9\%). Em um estudo de coorte, conduzido em 126 pacientes de um programa domiciliar na cidade de Belo Horizonte, Minas Gerais, a incidência de LPP foi de $20 \%$, sendo que $48,6 \%$ dos pacientes desenvolveram lesão na região sacral, seguida da região dos trocanteres e calcâneos, ambos com $19,2 \%{ }^{(24)}$. Outro estudo(15) conduzido em Belo Horizonte, Minas
Gerais, identificou uma prevalência de 12,8\% de LPP. Já em estudo da região do nordeste ${ }^{(5)}$, a prevalência de LPP foi de $24,9 \%$. Ressalta-se que as alterações fisiológicas decorrentes do processo de envelhecimento, a condição clínica dos pacientes da AD, bem como, fatores internos e externos (exposição à umidade, pressão contínua e prolongada sobre a pele e tecidos adjacentes, diminuição da sensibilidade, de alterações do metabolismo, estado nutricional, imunidade, dentre outros) podem contribuir, conjuntamente, para o aumento na frequência de LPP(24-25).

Quanto à assistência multiprofissional, observou-se que os profissionais mais atuantes foram o enfermeiro $(83,2 \%)$ e o médico (82,4\%). O profissional enfermeiro tem um papel essencial na assistência do paciente domiciliar, por elaborar e implementar o programa de cuidados, cabendo-lhe orientar, educar e supervisionar a assistência prestada(21). Já o médico destaca-se por ser o profissional responsável pela prescrição de medicamentos ${ }^{(5)}$

\section{Limitações do estudo}

Em relação às limitações do estudo, este é derivado de um estudo transversal, não sendo possivel investigar condições de baixa prevalência ou a inferência causal.

\section{Contribuições do estudo para a prática}

A partir do conhecimento do perfil clínico e epidemiológico dos pacientes atendidos por programa público de atenção domiciliar, pode-se favorecer o planejamento e implementação de intervenções acuradas com vistas à prevenção, resolução de problemas e promoção da saúde, evitando assim, internações ou complicações futuras.

\section{CONCLUSÃO}

A partir dos resultados encontrados, é possível verificar que a maioria dos pacientes eram idosos, do sexo feminino, restritos ao leito, com doença vascular, sendo o diagnóstico mais comum o AVE. O atendimento foi realizado principalmente pelos profissionais enfermeiro e médico.

A caracterização do perfil, além de descrever de forma clara e concisa problemas reais e potenciais, contribui para o cuidado do paciente, impactando no planejamento e implementação de intervenções adequadas.

Recomenda-se a investigação dos fatores associados aos diversos desfechos em pacientes atendidos em domicílio. Além disso, são necessários estudos que permitam estabelecer quais os melhores cuidados com vista à prevenção, promoção, tratamento e reabilitação das condições de saúde de pacientes atendidos em domicílio. 


\section{Contribuição dos autores}

Concepção e/ou desenho: Silva DVA e Araújo DD; Análise e interpretação dos dados: Silva DVA e Araújo DD; Redação do artigo e revisão crítica: Silva DVA, Carmo JR, Cruz MEA, Rodrigues CAO, Santana ET e Araújo DD; Revisão final: Araújo DD. 


\section{REFERÊNCIAS}

1. Sousa SPO, Branca SBP. Panorama epidemiológico do processo de envelhecimento no mundo, Brasil e Piaui: evidências na literatura de 1987 a 2009. Enferm Foco [Internet]. 2011 [cited 2018 Oct 31]:2(3):188-190. Available from: http://revista.cofen.gov.br/index.php/enfermagem/article/ view/132/113

2. Souza MFM, Malta DC, França EB, Barreto ML. Changes in health and disease in Brazil and its States in the 30 years since the Unified Healthcare System (SUS) was created. Ciênc. saúde coletiva [Internet]. 2018 [cited 2018 Jul 03]:23(6):1737-1750. Available from: http://www.scielo.br/pdf/csc/ v23n6/en_1413-8123-csc-23-06-1737.pdf

3. Fertonani HP, Pires DEP de, Biff D, Scherer MDA. The health care model: concepts and challenges for primary health care in Brazil. Ciênc. saúde coletiva [Internet]. 2015 [cited 2018 July 12];20(6):1869-1878. Available from: http://www.scielo.br/pdf/csc/v20n6/en_1413-8123-csc-20-06-1869.pdf

4. Silva YC, Silva KL, Brito MJM. Power relationships in home care: tensions and contradictions between professionals, users and caregivers. Rev enferm UFPE on line [Internet]. 2018 [cited 2018 July 04]:12(4):897-907. Available from: https://periodicos.ufpe.br/revistas/revistaenfermagem/ article/view/110272/28626

5. Carnaúba CMD, Silva TDA, Viana JF, Alves JBN, Andrade NL, Trindade Filho EM. Clinical and epidemiological characterization of patients receiving home care in the city of Maceió, in the state of Alagoas, Brazil Rev Bras Geriatr Gerontol [Internet]. 2017 [cited 2018 July 04];20(3):353363. Available from: http://www.scielo.br/pdf/rbgg/v2On3/1809-9823rbgg-20-03-00352.pdf

6. Brasil. Ministério da Saúde. Gabinete do Ministro. Portaria no 825, de 25 de abril de 2016. Redefine a Atenção Domiciliar no âmbito do Sistema Único de Saúde (SUS) e atualiza as equipes habilitadas. Brasilia: Ministério da Saúde [Internet]. 2016 [cited 2018 July 03]. Available from: http://bvsms. saude.gov.br/bvs/saudelegis/gm/2016/prt0825_25_04_2016.html

7. Silva KL, Silva YC, Lage EG, Paiva PA, Dias OV. Why is it better at home? Service users' and caregivers' perception of home care. Cogitare Enferm [Internet]. 2017 [cited 2018 July 04]:(22)4:e49660. Available from: https:// revistas.ufpr.br/cogitare/article/view/49660/pdf_en

8. Braga PP, Sena RR, Seixas CT, Castro EAB, Andrade MA, Silva YC. Oferta e demanda na atenção domiciliar em saúde. Ciênc. saúde coletiva [Internet] 2016 [cited 2018 July 04];21(3):903-12. Available from: http://www.scielo.br/ pdf/csc/v2In3/1413-8123-csc-21-03-0903.pdf

9. Castro EAB, Leone DRR, Santos CM, Neta FCCG, Gonçalves JRL, Contim D et al. Home care organization with the Better at Home Program. Rev Gaúcha Enferm [Internet]. 2018 [cited 2018 July 04]:39:e2016-0002. Available from: http://seer.ufrgs.br/index.php/RevistaGauchadeEnfermagem/article/ view/79526/46511

10. Brito MJM, Andrade AM, Caçador BS, Freitas LFC, Penna CMM. Home care in the structuring of the healthcare network: following the paths of comprehensiveness. Esc Anna Nery [Internet]. 2013 [cited 2018 July 04]:17(4):603-610. Available from: http://www.scielo.br/pdf/ean/v17n4/ en_1414-8145-ean-17-04-0603.pdf

11. Simão VM, Mioto RCT. O cuidado paliativo e domiciliar em paises da América Latina. Saúde Debate [Internet]. 2016 [cited 2018 July 04]:40(108)156-69. Available from: http://www.scielo.br/pdf/sdeb/ v40n108/0103-1104-sdeb-40-108-00156.pdf

12. Martelli DRB, Silva MS da, Carneiro JA, Bonan PRF, Rodrigues LHC Martelli-Júnior H. Internação domiciliar: o perfil dos pacientes assistidos pelo Programa HU em Casa. Physis [Internet]. 2011 [cited 2018 July 04]:21(1):147-57. Available from: http://www.scielo.br/pdf/physis/v2ln1/ v2lnla08.pdf

13. Duca GFD, Martinez AD, Bastos GAN. Perfil do idoso dependente de cuidado domiciliar em comunidades de baixo nivel socioeconômico de Porto Alegre, Rio Grande do Sul. Ciênc. saúde coletiva [Internet]. 2012 [cited 2018 July 04]:17(5):1159-65. Available from: http://www.scielo.br/pdf/ csc/v17n5/al0v17n5.pdf
14. Benassi V, Leandro J, Medeiros R, Taballi R. Perfil epidemiológico de paciente em atendimento fisioterapêutico em Home Care no Estado de São Paulo. J Health Sci Inst [Internet]. 2012 [cited 2018 July 04];30(4):3958. Available from: http://fisioterapia.com/wp-content/uploads/2017/02/ V30_n4_2012_p395a398.pdf

15. Biscione FM, Szuster DAC, Drumond EF, Ferreira GUA, Turci MA, Lima Júnior JF, et al. Avaliação de efetividade da atenção domiciliar de uma cooperativa médica de Belo Horizonte, Minas Gerais. Cad Saúde Pública [Internet]. 2013 [cited 2018 July 04];29(suppl 1):73-80. Available from: http://www.scielo.br/pdf/csp/v29sl/a07.pdf

16. Keenan G, Stocker J, Barkauskas V. Treder M. Heath C. Toward integrating a common nursing data set in home care to facilitate monitoring outcomes across settings. Journal of Nursing Measurement. [Internet]. 2003 [cited 2018 July 04]:7(2):74-83. Available from: https://www.ncbi.nlm.nih.gov/ pubmed/15274523

17. Asencio JMM, Herrera JCM, Santos FJM, Jimenez EG, Gallego MCF, Nieves $\mathrm{CB}$, et al. The association between nursing diagnoses, resource utilisation and patient and caregiver outcomes in a nurse-led home care service: Longitudinal study. International journal of nursing studies. [Internet] 2009 [cited 2018 July 04]:46(2):189-96. Available from: https:// www.sciencedirect.com/science/article/pii/S0020748908002526

18. Silva PAB, Santos FC, Soares SM, Silva LB. Sociodemographic and clinical profile of elderly persons accompanied by Family Health teams under the gender perspective. Rev Fund Care [Internet]. 2018 [cited 2018 July 04]:10(1):97-105. Available from: http://seer.unirio.br/index.php/ cuidadofundamental/article/view/5987/pdf

19. Moraes EN, Lanna FM, Santos RR, Bicalho MAC, Machado CJ, Romero DE. A new proposal for the clinical-functional categorization of the elderly: Visual Scale of Frailty (VS-Frailty). J Aging Res Clin Pract [Internet]. 2016 [cited 2018 July 12];5(1):24-30. Available from: http://www.jarcp.com/1808a-new-proposal-for-the-clinical-functional-categorization-of-theelderly-visual-scale-of-frailty-vs-frailty.html

20. Brondani MC, Ramos LH, Lampert M, Seiffert MA, Bruinsma JL, Beuter M. Caracterização de pacientes dependentes de tecnologias de um serviço de internação domiciliar. Rev Enferm UFSM [Internet]. 2013 [cited 2018 July 04]:3(Esp.):689-99.Available from: https://periodicos.ufsm.br/reufsm/ article/view/11063/pdf

21. Faria ACA, Martins MM, Schoeller SD, Matos LO. Care path of person with stroke: from onset to rehabilitation. Rev Bras Enferm [Internet]. 2017 [cited 2018 July 04];70(3):495-503. Available from: http://www.scielo.br/ pdf/reben/v70n3/0034-7167-reben-70-03-0495.pdf

22. Oliveira MVP, Buarque DC. Polypharmacy and the use of potentially inappropriate medications among aged inpatients. Geriatr Gerontol Aging [Internet]. 2018 [cited 2018 July 09]:12(1):38-44. Available from: http://docs. bvsalud.org/biblioref/2018/06/904988/gga-v12nl_in_38-44.pdf

23. Brasil. Ministério da Saúde, Secretaria de Atenção à Saúde. Caderno de Atenção Domiciliar: cuidados em terapia nutricional. Brasilia, DF: Ministério da Saúde [Internet]. 2015 [cited 2018 July 03]. Available from: http://189.28.128.100/dab/docs/portaldab/publicacoes/caderno_ atencao_domiciliar_vol3.pdf

24. Freitas JPC, Alberti LR. Application of the Braden Scale in the home setting: incidence and factors associated with pressure ulcers. Acta paul enferm [Internet]. 2013 [cited 2018 July 09];26(6):515-521. Available from: http://www.scielo.br/pdf/ape/v26n6/en_02.pdf

25. Moro JV. Caliri MHL. Pressure ulcer after hospital discharge and home care. Esc Anna Nery [Internet]. 2016 [cited 2018 July 04];20(3):e20160058. Available from: http://www.scielo.br/pdf/ean/v2On3/en_1414-8145ean-20-03-20160058.pdf 